\title{
Active Roles of Water in Aqueous Assembly of Macromolecules ${ }^{\dagger}$
}

\author{
Xiaosong Wang ${ }^{1, *}$ \\ 1 Department of Chemistry and Waterloo Institute for Technology, University of Waterloo, Waterloo, Canada, N2L 3G \\ * Correspondence: Xiaosong.wang @uwaterloo.ca (X.W.); \\ $\uparrow$ Presented at Materials Chemistry and Physics (Materials Chemistry 2020) - International e-Conference
}

Received: 16.09.2020; Revised: 20.09.2020; Accepted: 24.09.2020; Published: 27.09.2020

\begin{abstract}
Aqueous self-assembly customarily focuses on the molecular interactions of assembling building blocks; the role of water is barely studied. The hydration of hydrophobic $\mathrm{P}^{+} \mathrm{X}^{-}\left(\mathrm{P}^{+}\right.$: macromolecular phosphonium cation, $\mathrm{X}^{-}$: anion) is dependent on the ionic end groups, which is responsible for the consequent assembling behavior. The water interaction with the backbone was analyzed by FT-IR, and the dynamics were measured by low field-NMR spectroscopy. The combination of these two techniques reveals the effect of $\mathrm{X}^{-}$on hydration. When $\mathrm{X}^{-}$is $\mathrm{I}^{-}$, the ionic end group ordered water molecules that exerted a detectable long-range effect de-hydrating the backbone. The consequent hydrophobic interaction drove the aqueous assembly of $\mathrm{P}^{+} \mathrm{I}^{-}$into micelle-like aggregates with the ionic group exposed to water. In contrast, the ion pair with a hydrophobic anion of $\left[\mathrm{BPh}_{4}\right]^{-}$was not able to hold water and did not deplete the hydration water. The hydrated backbone of $\mathrm{P}^{+}\left[\mathrm{BPh}_{4}\right]^{-}$assembled into vesicles that were driven by hydration interactions. This elucidation at the molecular level is craved to progress aqueous supramolecular chemistry.
\end{abstract}

Keywords: aqueous assembly; hydrophobic effects; macromolecules.

(C) 2020 by the authors. This article is an open-access article distributed under the terms and conditions of the Creative Commons Attribution (CC BY) license (https://creativecommons.org/licenses/by/4.0/).

\section{Funding}

This research was funded by the Natural Sciences and Engineering Research Council of Canada (NSERC) [funding reference number 50503-10556].

\section{Acknowledgments}

This research has no acknowledgment.

\section{Conflicts of Interest}

The authors declare no conflict of interest. 